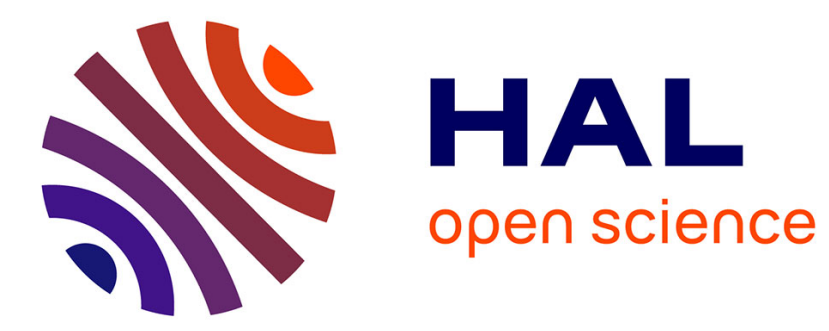

\title{
Emploi d'une liaison radioélectrique dans l'étude des coïncidences entre les impulsions provenant de compteurs séparés par de grandes distances \\ E. Picard, A. Rogozinski, M. Surdin
}

\section{- To cite this version:}

E. Picard, A. Rogozinski, M. Surdin. Emploi d'une liaison radioélectrique dans l'étude des coïncidences entre les impulsions provenant de compteurs séparés par de grandes distances. J. Phys. Radium, 1951, 12 (9), pp.854-859. 10.1051/jphysrad:01951001209085400 . jpa-00234497

HAL Id: jpa-00234497

https://hal.science/jpa-00234497

Submitted on 1 Jan 1951

HAL is a multi-disciplinary open access archive for the deposit and dissemination of scientific research documents, whether they are published or not. The documents may come from teaching and research institutions in France or abroad, or from public or private research centers.
L'archive ouverte pluridisciplinaire HAL, est destinée au dépôt et à la diffusion de documents scientifiques de niveau recherche, publiés ou non, émanant des établissements d'enseignement et de recherche français ou étrangers, des laboratoires publics ou privés. 


\title{
EMPLOI D'UNE LIAISON RADIOÉLEGTRIQUE DANS L'ÉTUDE DES GOÏNGIDENGES ENTRE LES IMPULSIONS PROVENANT DE GOMPTEURS SÉPARÉS PAR DE GRANDES DISTANGES
}

\author{
Par E. PICARD, A. ROGOZINSKI et M. SURDIN. \\ Commissariat à l'Énergie atomique, Fort de Châtillon, Fontenay-aux-Roses (Seine).
}

\begin{abstract}
Sommaire. - Examen des conditions d'emploi d'une liaison radioélectrique sans fil dans la sélection des coïncidences entre les décharges déclenchées par des gerbes atmosphériques dans des compteurs séparés par de grandes distances; description d'un ensemble de circuits permettant une telle sélection.

Dans le dispositif réalisé, destiné à l'étude de gerbes atmosphériques de très grande envergure, cet ensemble est associé à un circuit à coïncidences multiples (circuit à groupe maître) desservant deux ensembles de compteurs, jouant respectivement le rôle de compteurs maîtres et de compteurs analyseurs.

Le dispositif comprend ainsi, essentiellement :

I $^{\circ}$ un circuit maître à coïncidences triples, disposé à l'une des deux stations utilisées;

$2^{\circ}$ huit compteurs analyseurs, disposés à l'autre station;

$3^{\circ}$ un émetteur unique, transmettant les impulsions du circuit maître vers le récepteur placé à l'entrée des circuits analyseurs.

Deux genres d'émetteurs ont été mis au point : l'un, fonctionnant sur $5 \mathrm{Mc}: \mathrm{s}$, est destiné à des mesures où les aériens d'émission et de réception ne se trouvent pas en vision directe; l'autre, fonctionnant sur $10000 \mathrm{Mc}: \mathrm{s}$, est utilisé en terrain découvert; leur puissance est suffisante pour assurer une portée de $20 \mathrm{~km}$ environ.

Chaque circuit analyseur est pourvu d'un circuit à retard ajustable entre o,5 et i o $\mu \mathrm{s}$, rendant possible la compensation du retard que subit le signal maitre dans sa propagation entre les deux stations; il permet également l'étude des coïncidences retardées entre les décharges dans les différents compteurs. Le pouvoir de résolution du dispositif n'est limité, pratiquement, que par les retards statistiques intrinsèques de la décharge dans les compteurs utilisés.

Les premiers résultats obtenus montrent l'existence de coïncidences vraies sur une base de $600 \mathrm{~m}$ .et de $\mathrm{i} \mathrm{km}$.
\end{abstract}

1. Introduction. - Dans une Note récente ( $\left.{ }^{1}\right)$, nous avons donné une brève description d'un dispositif utilisant une liaison radioélectrique, destiné à sélectionner des cö̈ncidences entre compteurs éloignés. Un tel dispositif a été mis au point dans le but particulier d'effectuer des mesures concernant les gerbes atmosphériques de très grande envergure $\left({ }^{2}\right)$.

La liaison par câble, seule employée jusqu'à présent, entre compteurs éloignés, peut être réalisée d'une façon relativement aisée jusqu'à des distances de quelques centaines de mètres. Mais, dès que cette distance devient notablement plus grande, une telle liaison pose des problèmes d'ordre électrique et mécanique que seule une transmission radioélectrique permet de surmonter sans grandes difficultés.

Examinons sommairement quelques aspects du problème. Envisageons d'abord le cas le plus simple de deux stations, comprenant chacune un seul compteur. Pour étudier les coïncidences entre les impulsions provenant de ces compteurs, deux solutions sont à envisager, suivant que le dispositif de coïncidences est situé en dehors des stations, ou à l'une d'entre elles.

(1) C. R. Acad. Sc., 1951, 232, 503.

$\left({ }^{2}\right)$ A. Rogozinski, C. R. Acad. Sc., i 95 г , 232, $3{ }_{2} 8$.
Dans la première solution, chaque compteur est associé à un émetteur, fonctionnant sur une longueur d'onde bien déterminée. Dans le cas où il est possible de rendre l'émission suffisamment directive, les deux émetteurs pourraient fonctionner sur la même longueur d'onde. Sinon, les deux longueurs d'onde devraient être différentes. Le dispositif de coïncidence sera actionné par deux récepteurs détectant les signaux provenant respectivement de chaque station. Cette solution est susceptible d'être généralisée à un nombre quelconque de stations.

La seconde solution permet de simplifier le dispositif, par la suppression de l'un des émetteurs, ainsi que du récepteur correspondant. C'est cette solution que nous avons adoptée dans la première phase de nos recherches sur les gerbes atmosphériques.

En fait, on ne peut se borner au cas simple envisagé, car la fréquence très faible des phénomènes étudiés exige un nombre important de compteurs en coïncidence. De plus, l'intérêt d'étudier simultanément des gerbes de densité plus ou moins grande conduit à utiliser un dispositif à coïncidences multiples. A cet égard, le dispositif fondé sur le prin- 
cipe du " Groupe-Maître " $\left({ }^{3}\right)$ se prête bien au but recherché : il permet d'accroître dans une proportion considérable le nombre de coïncidences susceptibles d'être enregistrées en un temps déterminé.

Dans le présent travail, nous donnons une description détaillée du dispositif, réalisé sur ce principe, et dans lequel l'émetteur est associé au groupe des compteurs maitres et le récepteur au groupe des compteurs analyseurs.

Enfin, pour que le rapport entre les coïncidences fortuites et les coincidences vraies devienne acceptable, le temps de résolution des circuits de coïncidences a été réduit au minimum, compatible avec l'emploi des compteurs G. M. du type utilisé; ce temps minimum a été déterminé dans une étude préalable, qui fera l'objet d'une publication ultérieure.

\section{Ghoix de la longueur d'onde et de la bande} passante de l'émission radioélectrique. - Ce sont la bande passante et la portée de l'émetteur, de même que des considérations concernant l'encombrement du matériel nécessaire, qui déterminent principalement le choix de la longueur d'onde. De plus, ce choix doit être en accord avec les réglements administratifs régissant l'emploi des émissions radioélectriques. Seule l'utilisation d'ondes centimétriques est exempte de telles restrictions. Enfin, une autre considération entre en ligne de compte dans ce choix : celle de l'indépendance de la réception vis-à-vis d'une émission brouilleuse. Cette dernière condition peut, en particulier, être satisfaite en rendant l'émission suffisamment directive.

En ce qui concerne la bande passante, elle est conditionnée par la forme du signal de modulation, qui provient, à l'origine, des compteurs G. M. Étant donné la faible valeur du temps de résolution $(<\mathrm{r} \mu \mathrm{s})$, imposée par la nature des mesures, l'émission doit être capable de transmettre des phénomènes transitoires rapides de l'ordre de $0, \mathbf{I} \mu \mathrm{s}$. La largeur de la bande passante correspondante doit donc être au moins égale à $4 \mathrm{Mc}: \mathrm{s}$.

D'autre part, l'émission doit assurer la liaison entre les stations, lorsque la distance qui les sépare est comprise entre une cinquantaine de mètres et plusieurs kilomètres. Ce problème se résout sans difficulté par l'emploi d'un émetteur de faible puissance, fonctionnant sur une longueur d'onde décamétrique ou inférieure.

Les diverses conditions examinées, concernant la bande passante et la portée de l'émetteur, ainsi que l'indépendance de la réception vis-à-vis d'une émission brouilleuse, se trouveront réalisées, si l'on a recours à une liaison fonctionnant sur ondes centimétriques. Aussi, avons-nous choisi une longueur d'onde comprise dans la bande usuelle des $3 \mathrm{~cm}$.

(') A. Rogozinski, Ann. Phys., 1945, 20, 39ı.
Le dispositif ainsi réalisé peut fonctionner indifféremment en plaine ou en montagne. Cependant, les aériens de l'émetteur et du récepteur correspondant doivent être placés en vision directe $\left({ }^{4}\right)$.

3. Description du dispositif. Gas simple. Pour faciliter la description du dispositif utilisé, reprenons le cas de deux stations $\mathrm{E}$ et $\mathrm{R}$, pourvues, chacune, d'un seul compteur G. M. Tous les circuits partiels, qui y sont associés, se retrouvent, d'ailleưrs, dans le dispositif complet.

A. Principe. - Le problème consiste à sélectionner les coïncidences entre les décharges du compteur placé à la station émettrice $\mathrm{E}$ et les
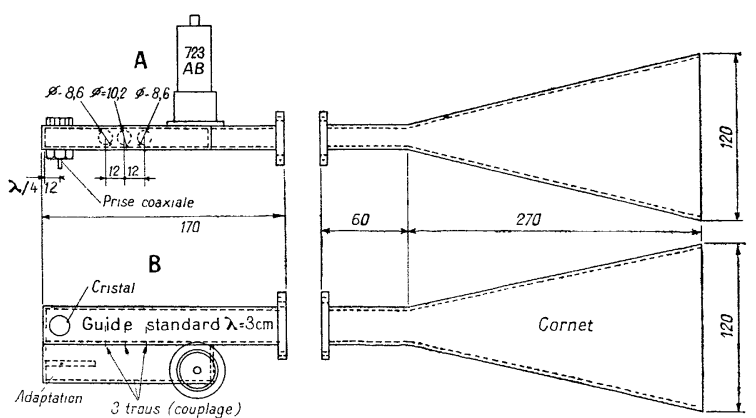

Fig. I - Tête haute-fréquence de réception.

Une vue de côté (A) et une vue de dessus (B) montrent la disposition des éléments de la tête haute-fréquence du récepteur. Sur la figure $B$, en particulier, on distingue le guide d'ondes principal et le guide d'ondes auxiliaire qui, couplé au premier par les trois trous d'un coupleur directionnel, alimente le cristal sur lequel se fait le changement de fréquence.

Le cornet du récepteur, figurant sur les dessins A et B, est identique au cornet d'émission.

décharges du compteur " local " de la station réceptrice R. Le schéma de principe du dispositif correspondant est celui de la figure 3, qui ne comprendrait, cependant, qu'un seul compteur à chaque station $\mathrm{E}$ et R.

Les impulsions délivrées par chaque compteur sont amplifiées et mises en forme par un préamplificateur.

A la station E, l'impulsion déclenche un monovibrateur dont le signal, de forme bien déterminée, module l'émetteur.

A la station $R$, un récepteur démodule le signal qui lui parvient. Le signal démodulé est ensuite mis en forme et appliqué au circuit de coïncïdence. Le signal provenant du compteur local est envoyé dans le même circuit de coïncidence, après avoir été retardé, dans un circuit approprié, d'une quantité

$\left({ }^{4}\right)$ Dans le cas où il n'est pas possible de le faire, il convient d'avoir recours à un ensemble qui n'est pas sujet à une telle restriction. Nous avons mis au point, dans ce but, un dispositif fonctionnant sur $5 \mathrm{Mc}: \mathrm{s}$. 
égale au temps de propagation du premier signal entre les deux stations $\mathrm{E}$ et $\mathrm{R}$.

B. Émetteur. - L'émetteur est constitué essentiellement par un klystron reflex $723 \mathrm{~A}-\mathrm{B}$, accordé sur une fréquence $f$ voisine de $\mathrm{ro}$ ooo $\mathrm{Mc}: \mathrm{s}$, alimentant un guide d'ondes terminé par un cornet. En l'absence de la modulation, le klystron est au repos. Son oscillation est provoquée par le signal rectangulaire, délivré par le modulateur, décrit plus loin, et appliqué sur l'anode réflectrice du klystron. La puissance rayonnée est de $30 \mathrm{~mW}$ environ. Les dimensions du cornet, indiquées sur la figure $\mathrm{I}$, et la sensibilité du récepteur utilisé permettent de réaliser une portée de l'ordre de $20 \mathrm{~km}$, suffisante pour nos recherches actuelles.

C. Récepteur. - La partie haute-fréquence du récepteur est constituée par un cornet, identique à celui de l'émetteur, alimentant le guide d'ondes principal, dans lequel se trouve placé le cristal détecteur ( $f i g$. I). L'oscillateur local, un klystron $723 \mathrm{~A}-\mathrm{B}$, est accordé sur la fréquence $f+3 \mathrm{o} \mathrm{Mc}: \mathrm{s}$ et rayonne dans un guide auxiliaire adapté. Il est couplé au guide d'ondes principal par un coupleur directionnel à trois trous, de sorte que les ondes locales ne se propagent que dans la direction du cristal détecteur et avec l'affaiblissement désiré; c'est aux bornes de ce cristal que le préamplificateur moyenne fréquence recueille le signal. L'ensemble, constitué par le préamplificateur et l'amplificateur moyenne fréquence, est accordé sur 3 o Mc : s. Son gain total est de l'ordre de roo db et sa bande passante de $6 \mathrm{Mc}: \mathrm{s}$ environ. On a choisi une bande passante plus large qu'il n'était nécessaire, afin d'éviter l'emploi d'un contrôle automatique de fréquence.

Après amplification, le signal moyenne fréquence est détecté et le signal détecté déclenche un monovibrateur du type rapide, décrit plus loin, qui le met en forme. Le signal rectangulaire, délivré par le monovibrateur, a une durée qui peut être réglée à volonté entre $o, 3$ et $5 \mu \mathrm{s}$. Ce signal est finalement appliqué au circuit de coïncidences.

D. Circuit de coincidences. - Le circuit employé est un circuit de Rossi. Pour les applications envisagées, le temps de résolution du dispositif doit être réduit dans la mesure du possible. Toutefois, sa valeur minimum est moins déterminée par les circuits électroniques que par les retards intrinsèques de réponse des compteurs G. M.

Une étude particulière des retards relatifs entre les impulsions, provenant de plusieurs compteurs déchargées par la même particule ionisante, nous a montré que 9o pour ıoo de ces impulsions présentaient entre elles des retards compris dans une bande ayant une largeur de $0,4 \mu$ s. Cette valeur n'est naturellement valable que pour le type de compteurs utilisés.
Nous avons adopté comme temps de résolution $\tau=0,5 \mu \mathrm{s}$, estimant comme acceptable la faible perte d'efficacité qui résulte de ce choix. Un tel temps de résolution a été obtenu, en donnant aux signaux appliqués au circuit de coïncidences une forme convenable et à la constante de temps du circuit plaque de Rossi une valeur de $o,\{\mu \mathrm{s}$.

On trouvera, dans le travail de M. G. Valladas $\left(^{5}\right)$, une discussion détaillée de l'influence de ces facteurs sur le temps de résolution.

La valeur de $:$ peut être réglée entre 0,12 et $5 \% \mathrm{~s}$, en agissant sur la durée du signal provenant du compteur éloigné (à l'aide du circuit de mise en forme, signalé précédemment), le signal provenant du compteur local conservant toujours une durée de 0,3 i $\mathrm{s}$.

E. Préamplificateur des compteurs G. M. - Comme nous l'avons vu, chaque compteur G. M. est suivi d'un préamplificateur, fixé directement sur lui.

L'emploi du préamplificateur répond aux deux buts suivants :

${ }^{\circ}{ }^{\circ}$ augmenter la sensibilité des circuits de coïncidences;

$2^{0}$ mettre en forme les impulsions des compteurs.

Chaque préamplificateur comporte deux étages d'amplification, équipés de pentodes $6 \mathrm{AH} 6$ (voir schéma d'ensemble, fig. 4). L'effet de self, dû à une surcompensation de la charge du premier étage, provoque une surtension de forme pratiquement invariable, dès que l'impulsion du compteur dépasse un certain seuil. Cette surtension est seule amplifiée par le second étage, dont la lampe, normalement polarisée au delà du cut-off, fonctionne avec une faible charge de plaque (200 $\Omega$ ). L'impulsion, dont l'amplitude est alors égale à $\check{\mathrm{V}} \mathrm{V}$ et la durée à $o, 3 \mu \mathrm{s}$, est transmise au préamplificateur par l'intermédiaire d'un câble à plusieurs conducteurs, lequel amène, par ailleurs, toutes les tensions d'alimentation.

F. Monovibrateur. - Ce circuit est employé à diverses reprises dans l'appareillage décrit. Déclenché par une impulsion dont l'amplitude doit être supérieure à $5 \mathrm{~V}$, il délivre un signal rectangulaire, de forme et de durée bien déterminées. Deux variantes du montage classique à réaction plaque-grille ont été mises au point.

Un monovibrateur rapide ( $f i g .{ }_{2}$ ) délivre un signal dont les fronts ont des pentes de l'ordre de $6.10^{8} \mathrm{~V}: \mathrm{s}$, sensiblement indépendantes de la durée totale du signal. Cette durée est déterminée par la constante de temps $\left(R_{g}^{\prime}+R_{p}\right) C$, à condition que $R_{g} C^{\prime} \gg\left(R_{g}^{\prime}+R_{p}\right) C$. Elle varie de o,3 à ı $2 \mu \mathrm{s}$, lorsqu'on fait varier $R_{g}^{\prime}$ de 4,5 à $200 \mathrm{k} \Omega$.

Un monovibrateur d'enregistrement (voir schéma

(5) J. Physique Rad., I951, 12, 53 A. 
d'ensemble, fig. 5) provoque l'allumage d'une lampe à néon dont l'éclair doit avoir une durée excédant i ms pour être enregistré aisément. Cet éclair est photographié et indique une coïncidence.

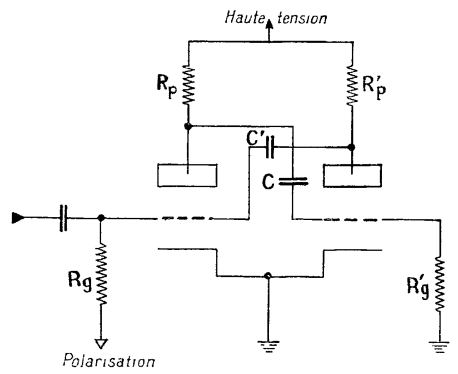

Fig. 2. - Monovibrateur rapide.

Le circuit temporisateur est constitué par les résistances $\left(R_{g}^{\prime}+R_{p}\right)$, en série avec la capacité $C$. La durée du signal rectangulaire peut être rendue variable, entre certaines limites, en agissant sur la valeur de $R_{r}^{\prime}$.

G. Circuit de retard. - Ce circuit est destiné à compenser le retard $t_{r}$ subi par le signal provenant du compteur éloigné, lors de sa propagation vers la station réceptrice $R$ : cette compensation est obtenue en retardant d'une valeur égale le signal provenant du compteur local. Ainsi, en cas de décharge simultanée des deux compteurs, leurs impulsions respectives se présenteront simultanément à l'entrée du circuit de coïncidences, situé à la station réceptrice. Le circuit de retard (voir le schéma d'ensemble, fig. 5) se compose, d'une part, d'un monovibrateur rapide dont la durée, ajustable, du signal négatif qu'il délivre est rendue égale à $t_{r}$. et, d'autre part, d'un étage de dérivation et d'amplification. Ce dernier étage amplifie seulement le signal positif résultant de la dérivation du signal rectangulaire fourni par le monovibrateur. La forme du signal dérivé reste invariable, quelle que soit la valeur de $t_{r}$. Après amplification, le signal dérivé a une amplitude d'une vingtaine de volts et une durée, à la base, de $o, 3 \mu \mathrm{s}$. Il est appliqué au circuit de coïncidence dont la lampe correspondante est bloquée par une polarisation grille de $4, \tilde{\partial} \mathrm{V}$, soit environ le cinquième de l'amplitude du signal délivré dont l'effet devient comparable à celui d'une impulsion légèrement trapézoïdale.

H. Modulateur. - Rappelons que le klystron oscille pendant la modulation. La tension de l'anode réflectrice, à laquelle est appliqué le signal de modulation, doit demeurer, pendant la durée de l'oscillation, aussi constante que possible, afin d'éviter toute modulation parasite de fréquence. C'est pourquoi, après un monovibrateur rapide, le modulateur comporte un étage d'amplification saturé (voir schéma d'ensemble, fig. 4).

4. Description du dispositif utilisé. - Rappelons d'abord brièvement les propriétés essentielles de la méthode du Groupe Maître :

Les impulsions provenant des compteurs analyseurs ne peuvent être transmises par les circuits associés que si elles se produisent simultanément avec une impulsion provenant des compteurs maîtres. Ce n'est que lorsqu'une telle coïncidence se produit que l'impulsion d'un compteur analyseur peut se traduire par un signal, qui, dans le cas présent, est l'éclair d'une lampe à néon. Tous ces éclairs sont enregistrés sur un film dont le dépouillement fait connaître les compteurs analyseurs, quel qu'en soit le nombre, qui ont été déchargés, simultanément, en coïncidence avec les compteurs maîtres.

Dans le dispositif réalisé, le compteur unique de
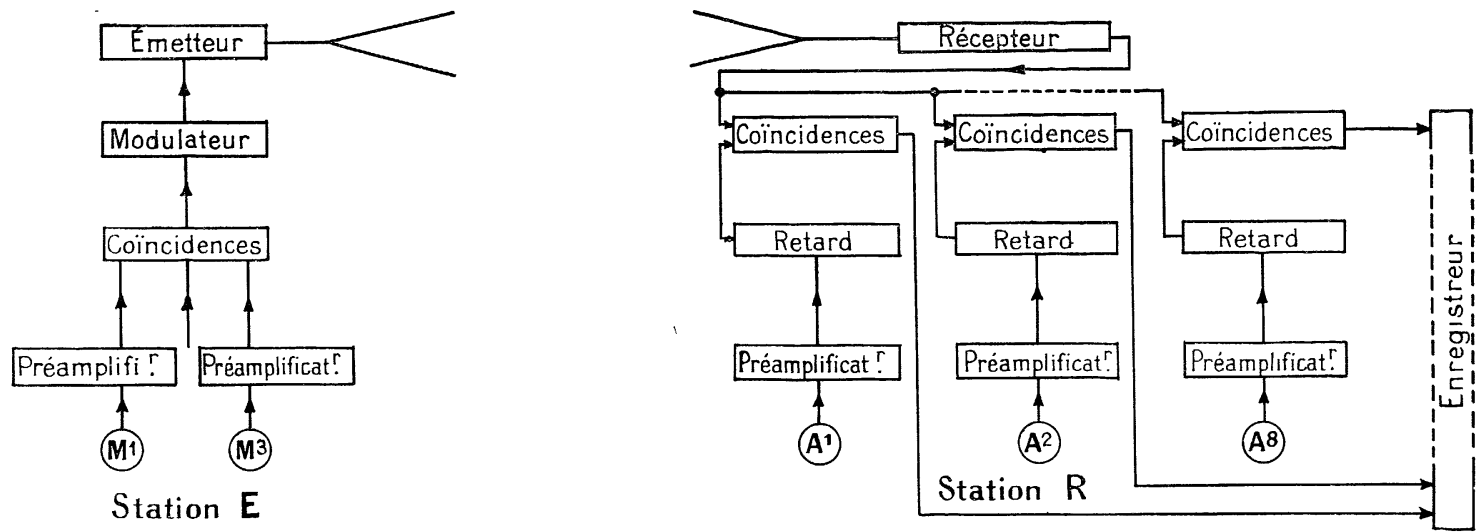

Fig. 3. - Schéma de principe du dispositif.

A la station E, le signal maître, délivré par le circuit à coïncidences triples, module l'émetteur. Le récepteur de la station $R$ reçoit, amplifie et démodule le signal maître. Après mise en forme, le signal démodulé débloque les huit circuits analyseurs, permettant ainsi aux signaux analyseurs, en coïncidence avec lui, d'être enregistrés.

Dans chaque circuit analyseur, un circuit de retard permet de compenser le retard subi par le signal maitre, lors de sa propagation entre les deux stations. 


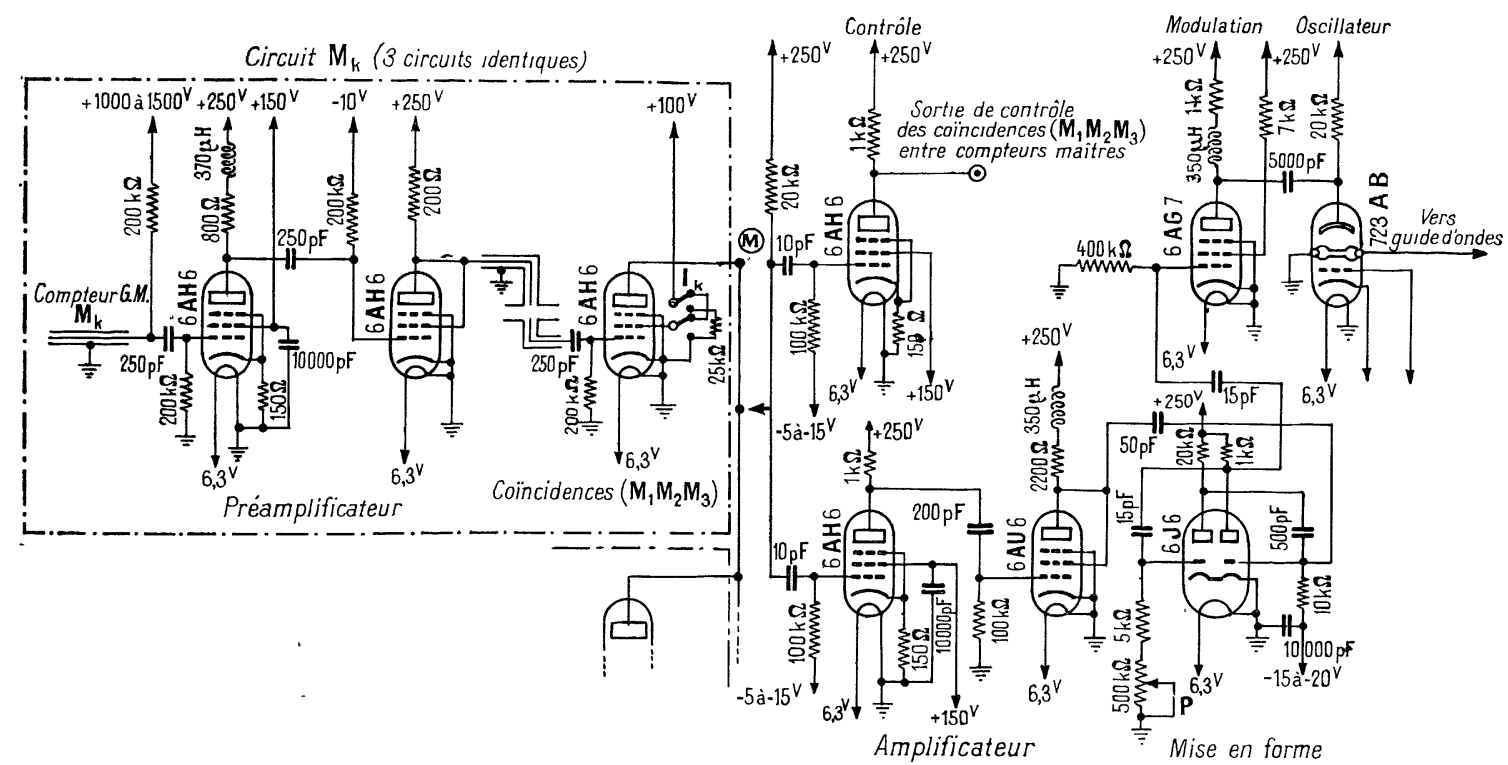

Fig. 4. - Schéma des circuits de la station E.

Le circuit maître comprend trois circuits identiques $\mathrm{M}_{L}(k=\mathrm{I}, 2,3)$ comportant, chacun, un préamplificateur et une lampe de coïncidence. L'interrupteur $I_{k}$ permet de supprimer du circuit de coïncidence le compteur $\mathrm{M}_{t}$, en bloquant la lampe de coïncidence correspondante.

Le signal maître $M$ est amplifié, mis en forme (la durée du signal est fixée par le potentiomètre $P$ ) et module finalement l'émetteur. Un étage de contrôle permet de vérifier la bonne marche de l'ensemble du circuit maître.

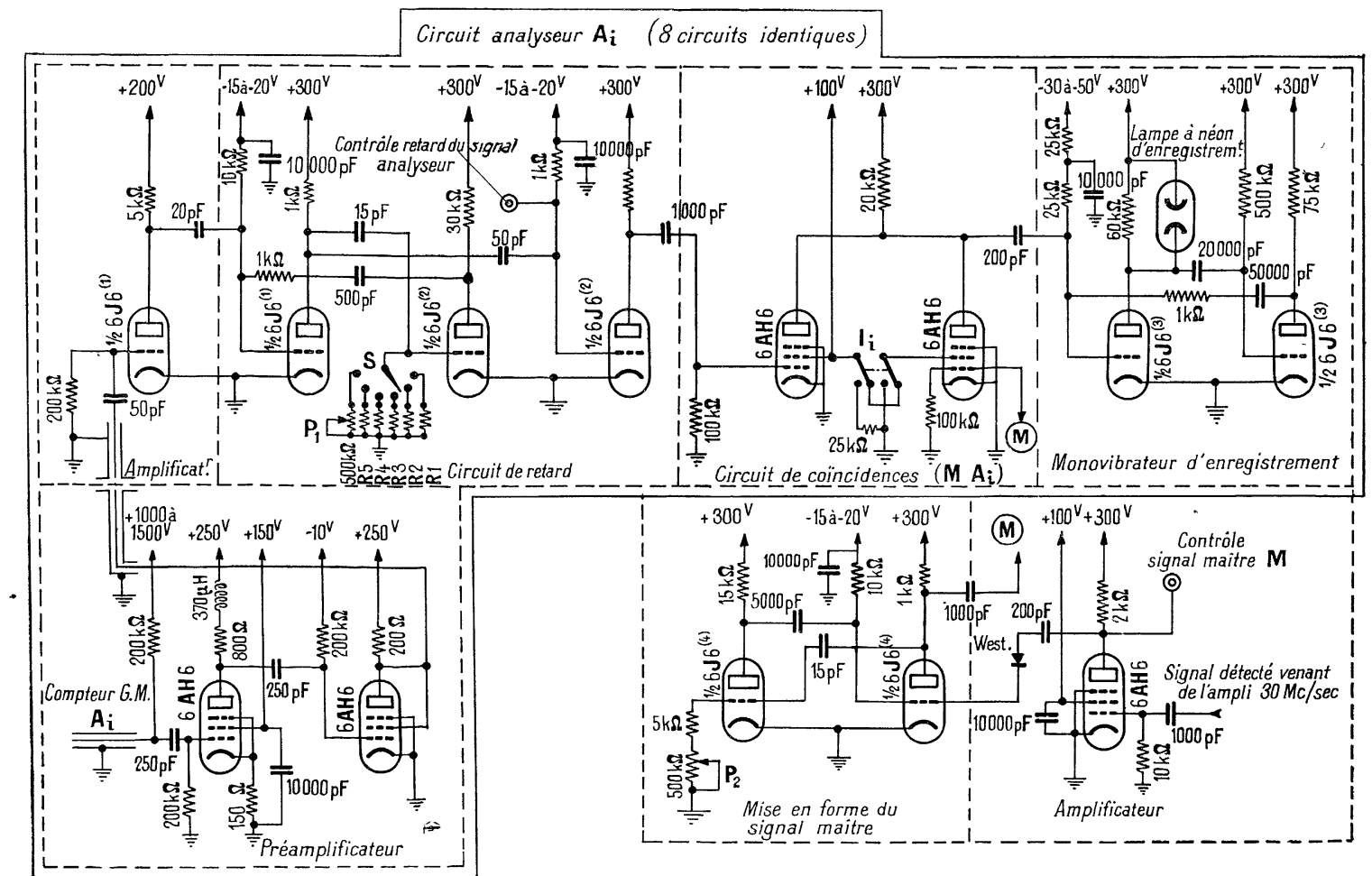

Fig. 5. - Schéma des circuits de la station R.

Le récepteur, du type superhétérodyne, n’est pas représenté sur ce schéma. A la sortie du récepteur, le signal M est mis en forme, sa durée pouvant être ajustée par le potentiomètre $\mathrm{P}_{2}$; le signal maître négatif est alors envoyé dans chacun des circuits de coïncidences (M, A) $l=8$ dont il bloque la lampe de Rossi correspondante (l'interrupteur $I_{\imath}$ permet, d'ailleurs, dans un but de contrôle de tout le circuit analyseur, de bloquer artificiellement cette lampe en mettant son écran à la masse). Le signal maître commande ainsi le passage du signal analyseur vers l'enregistreur. Au préalable, le signal analyseur a subi un retard, fixé par la position du potentiomètre $P_{1}$. Huit circuits identiques $A_{\text {, }}$, comprenant chacun un préamplificateur, un amplificateur, un circuit de retard, un circuit de coïncidence et un monovibrateur d'enregistrement, composent avec le circuit maître, situé à la station $\mathrm{E}$, l'ensemble du dispositif à coïncidences multiples, ou "Groupe Maître ". 
chaque station est remplacé par des groupes de compteurs, le groupe des compteurs maîtres étant disposé à la station $\mathrm{E}$ et le groupe des compteurs analyseurs à la station $\mathrm{R}$ ( fig. 3). Le circuit maître (voir schéma d'ensemble, fig. 4) est un circuit à coïncidences triples dont le signal est transmis à la station $\mathrm{R}$.

Le groupe analyseur est composé de huit compteurs; chaque compteur commande un circuit analyseur comprenant : un préamplificateur, un circuit de retard, un circuit à coïncidences doubles et un monovibrateur d'enregistrement (fig. 5). L'ensemble des huit circuits de coïncidence doubles n'est débloqué que par le signal maître. Celui-ci, symbolisé par la lettre $M$, peut représenter, suivant les cas, une coïncidence triple, double ou une impulsion simple provenant d'un seul compteur maître. L'enregistrement ainsi obtenu permet de dénombrer les coïncidences entre M, d'une part, et toutes les combinaisons des impulsions provenant des huit compteurs analyseurs, d'autre part.

Les circuits partiels utilisés dans ce dispositif sont identiques à ceux décrits dans le paragraphe 3 . Signalons, cependant, que le modulateur est maintenant précédé de deux étages d'amplification, qui définissent le front de montée du signal maître avec une précision supérieure à $\mathrm{o}, \mathrm{I} \mu \mathrm{s}$.

5. Premiers résultats. - Le tableau résume les premiers résultats obtenus à basse altitude pendant I $_{2} 3 \mathrm{~h}$ d'enregistrement à l'aide de l'appareillage décrit. Le dispositif particulier utilisé est schématisé par la figure 6, où la disposition des compteurs est apparente.

Signalons, en outre, qu'un certain nombre de mesures ont été effectuées sur une base de $1 \mathrm{~km}$, où quelques rares coïncidences vraies ont été observées; mais ces mesures n'ont pas été poursuivies, car l'un des groupes de compteurs n'a pu être disposé sous une couche absorbante suffisamment réduite.
Au cours d'un fonctionnement continu de plusieurs semaines, nous avons pu contrôler la bonne marche $\mathrm{du}$ dispositif décrit.

Station E

Station R
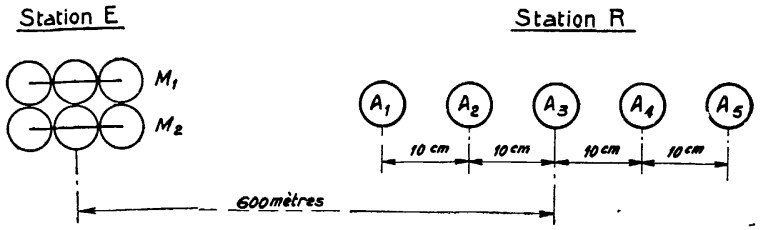

Fig. 6. - Disposition schématique des compteurs.

La surface utile de chacun des deux plateaux $M_{1}$ et $M_{2}$ du Groupe Maitre et de chacun des compteurs analyseurs A est respectivement égale à i 500 et $250 \mathrm{~cm}^{2}$.

TABLEAU.

Résultats pour une distance de $600 \mathrm{~m}$ entre les stations $\mathrm{E}$ et $\mathrm{R}(f i g .6)$.

La fréquence des impulsions délivrées par chacun des compteúrs analyseurs A est de 550 par minute, celle des coïncidences $\mathbf{M}=\mathbf{M}_{1} \mathbf{M}_{2}$ du groupe maître de 900 par minute. Les symboles $\mathbf{M}[\mathbf{A}]^{n}$, où $n=\mathrm{I}, 2, \ldots, 5$, représentent les coüncidences entre le signal M, d'une part, et au moins $n$ compteurs quelconques parmi les cinq compteurs analyseurs A utilisés, d'autre part. Dans les fréquences indiquées, on a déjà tenu compte des coïncidences fortuites pour chaque type de coïncidences.

\begin{tabular}{|c|c|c|c|c|c|}
\hline & $\mathrm{M}[\mathrm{A}]$. & $\mathrm{M}[\mathrm{A}]^{2}$. & $M[A]^{\prime}$. & $M[A]^{\prime}$. & $M[\boldsymbol{A}]^{5}$ \\
\hline $\begin{array}{c}\text { Nombre total de } \\
\text { coïncidences. }\end{array}$ & 173 & 18 & 8 & 5 & I \\
\hline Coïncidences par & & & & & \\
\hline 24 h......... & 33,6 & 3,6 & I, 6 & I & 0,2 \\
\hline
\end{tabular}

Nous tenons à remercier M. P. Goujon pour son concours actif dans la construction et la mise au point des différents circuits que comporte ce dispositif. 